\title{
Philosophiques
}

\section{Entrevue avec M. Matthew Lipman}

Volume 12, numéro 2, automne 1985

URI : https://id.erudit.org/iderudit/203292ar

DOI : https://doi.org/10.7202/203292ar

Aller au sommaire du numéro

Éditeur(s)

Société de philosophie du Québec

ISSN

0316-2923 (imprimé)

1492-1391 (numérique)

Découvrir la revue

Citer ce document

(1985). Entrevue avec M. Matthew Lipman. Philosophiques, 12(2), 393-409.

https://doi.org/10.7202/203292ar

Ce document est protégé par la loi sur le droit d'auteur. L'utilisation des services d'Érudit (y compris la reproduction) est assujettie à sa politique d'utilisation que vous pouvez consulter en ligne.

https://apropos.erudit.org/fr/usagers/politique-dutilisation/
Cet article est diffusé et préservé par Érudit.

Érudit est un consortium interuniversitaire sans but lucratif composé de l’Université de Montréal, l'Université Laval et l'Université du Québec à Montréal. Il a pour mission la promotion et la valorisation de la recherche. https://www.erudit.org/fr/ 


\section{INTERVENTIONS}

\section{ENTREVUE AVEC M. MATTHEW LIPMAN}

Depuis ses origines, la rubrique «Interventions » s'était limitée à publier des textes écrits ou à reproduire des discours prononcés lors d'événements importants. Lors d'une récente réunion du comité de rédaction de la revue a été proposée et acceptée l'idée d'inclure dorénavant des entrevues avec des personnalités marquantes du domaine philosophique. On trouvera ci-après le premier résultat de cette nouvelle politique, un entretien avec M. Matthew Lipman.

M. Lipman n'a guère besoin de présentation. Auteur d'un programme original d'enseignement de la philosophie aux enfants, il est aussi directeur d'un institut de philosophie, également pour enfants, qu'il a fondé à Montclair State College, au New Jersey. Depuis quelques années, ses travaux ont suscité beaucoup d'intérêt au Québec. En particulier, depuis plus d'un an déjà, la CEPH (Comité pour l'Enseignement de la Philosophie) de la Société de Philosophie du Québec en a poursuivi une analyse et une critique dont on peut trouver régulièrement le compte rendu dans le Bulletin de la Société.

Lors d'une rencontre, le 27 février à Montréal, entre les membres du CEPH et Matthew Lipman, M. Pierre Cohen-Bacrie, président de la S.P.Q. et membre du CEPH, a conduit l'entrevue dont le texte suit. Madame Judy Kyle a bien voulu, au cours de l'entretien, traduire les questions du français à l'anglais. Pierre Cohen-Bacrie a assuré pour publication la traduction de l'anglais au français des réponses de M. Lipman, qui les a luimême revues et corrigées. 
Philosophiques est donc heureuse, pour ce coup d'envoi, de faire une place à la fois aux travaux du CEPH et aux recherches novatrices de M. Lipman.

Nous avons fait suivre l'entretien d'une brève bibliographie. Ceux qui désireraient obtenir plus ample information peuvent ou bien écrire à l'adresse qui y est indiquée ou bien communiquer avec $M$. Gérard Potvin, président du CEPH, à l'adresse de la S.P.Q.

$$
\text { ** }
$$

CEPH. Monsieur Lipman, votre méthode consiste à intéresser les enfants par une nouvelle contenue dans le livre de l'élève (par exemple : la découverte d'Harry) et à engager une discussion où les enfants soulignent les idées qui les intéressent, et à traiter ces idées selon des explications et des exercices prévus dans le livre du mâtre.

$\grave{A}$ votre avis, la discussion que les enfants vont avoir entre eux doit-elle avoir un rapport direct avec le texte de la nouvelle et avec le livre du maître ? Et pensez-vous qu'il est souhaitable que l'enseignant intervienne dans la discussion des enfants pour faire des liens avec le texte de la nouvelle et le livre du maître? MATTHEW LIPMAN. Il doit y avoir une combinaison des deux éléments : d'une part, les discussions des enfants entre eux, d'autre part, les interventions de l'enseignant. L'enseignant doit faire montre de flexibilité, encourager les enfants à déterminer les sujets de discussion, puis utiliser les exercices du manuel afin d'être davantage en mesure de traiter les sujets particuliers choisis par les enfants et de renforcer les capacités des enfants. À condition cependant que l'enseignant n'utilise ces exercices qu'au moment où il semble que leur besoin se fasse sentir, comme un supplément mais non dans la perspective de contrôler le déroulement de la discussion. De telle sorte que les exercices s'adaptent à la discussion des enfants plutôt que la discussion des enfants à la structure du manuel d'instructions.

En somme, l'enseignant devrait pouvoir en quelque sorte compléter la discussion quand il a l'impression que les enfants ont dit ce qu'ils avaient à dire sur un chapitre particulier. Si; 
par ailleurs, l'enseignant est d'avis que les enfants n'ont pas souligné un élément important d'un chapitre, il (ou elle) pourrait alors suggérer de discuter de cet élément.

Ainsi, je ne dirais pas que l'enseignant ne doive pas intervenir mais que les enfants devraient considérer le texte de la nouvelle comme leur appartenant. C'est à partir de leur appropriation de la nouvelle que les enfants travaillent et c'est à cette condition que l'enseignant peut intervenir de façon constructive. Notons que le texte de la nouvelle est écrit de telle façon qu'il ne leur paraisse pas provenir d'un point de vue d'adulte.

CEPH. Dans cette perspective de l'appropriation de la nouvelle par les enfants, trouvez-vous pertinent que la discussion soit menée, animée, par un enfant qui serait volontaire pour le faire?

M.L. J'ai vu des enfants qui, dès le premier contact avec le programme, développent une telle assurance en observant la façon de procéder de l'enseignant qu'ils sont en mesure de conduire eux-mêmes la discussion. Quant à la question de savoir si une telle pratique est souhaitable dans le sens où elle deviendrait une façon régulière de procéder ou, en tout état de cause, tendrait à remplacer l'enseignant, c'est évidemment une tout autre histoire.

CEPH. Au niveau de la mise en œuvre de votre programme, considérez-vous qu'il est possible ou qu'il est souhaitable de laisser les enfants aller jusqu'au bout d'une discussion qui peut, parfois, s'étaler sur plusieurs heures, plusieurs semaines, sans intervenir dans le but de faire des liens, comme on le disait tout à l'heure, avec le texte de la nouvelle par exemple ? Cette question porte sur la pondération, c'est-à-dire sur le temps qui peut être consacré à un aspect de votre méthode plutôt qu'à un autre.

M.L. Je pense qu'il est possible d'aller assez loin dans cette direction. Le matériel a une certaine élasticité, de telle sorte que l'important n'est pas de couvrir l'ensemble, ce n'est pas la quantité mais la qualité qui importe. Si les discussions sont réellement fécondes, exploratoires et progressent de façon significative sur des questions d'ordre philosophique, je suppose qu'il sera intéressant de poursuivre ces discussions pendant un bon moment, des semaines peut-être. 
CEPH. Oui, mais iriez-vous jusqu'à dire que l'on pourrait ne pas finir la lecture de la nouvelle? Par exemple, pourrait-on se contenter, en tout et pour tout de quatre chapitres (sur les dixsept que compte la nouvelle Harry) parce que les discussions se sont prolongées?

M.L. Oui, je dirais que oui. Tout d'abord, il faut se rappeler que chaque nouvelle donne lieu à un programme étalé sur deux années. Un enseignant a donc comme matériel, pour une année, environ huit chapitres sur dix-sept. Ceci dit, nous voudrions que l'on s'assure que certains éléments, plus importants que d'autres soit parce qu'ils participent d'un ensemble cohérent comme la démarche logique dans Harry (c'est ainsi qu'il me semble impossible de ne pas traiter de certaines étapes pour arriver au syllogisme), soit parce que, et c'est le cas du dernier chapitre, ils concernent la partie de la nouvelle où un personnage exprime son scepticisme, critique l'ensemble de la démarche - que l'on s'assure donc que ces éléments soient vus.

Comment écarter cet aspect leibnizien que constitue la prise de conscience, chez les enfants, que l'objectivité peut être considérée comme le résultat d'un nombre infini de perspectives différentes qui sont interchangeables? C'est ainsi que l'on ne voudrait pas faire l'économie de cette prise de conscience qui, cependant, ne conduit pas au relativisme.

D'un autre côté, il y a effectivement des aspects de la nouvelle qui peuvent être sacrifiés, s'il le faut. Il m'est arrivé de recevoir une demande anxieuse de la part d'un responsable scolaire qui voulait savoir si quelques chapitres pouvaient ne pas être vus. Je n'ai éprouvé aucune difficulté à calmer son anxiété en lui répondant que c'était le cas. C'est finalement une question de jugement.

CEPH. Je souhaiterais aborder maintenant des questions plus théoriques ou philosophiques, si vous le permettez. Quel est le rôle que jouent les idées platoniciennes dans Harry ou dans Pixie? Par exemple, le questionnement sur la réalité des idées dans Harry, peut-on penser qu'il est inspiré philosophiquement du platonisme?

M.L. Manifestement, le dernier chapitre de Pixie est inspiré du Parménide et de La République de Platon; et les chapitres pré- 
cédents sont inspirés d'Empédocle, d'Héraclite etc. Manifestement, également, des emprunts ont été faits à l'aristotélisme dans Harry ; il n'y a rien là de surprenant puisqu'il y est question de la logique, du syllogisme par exemple. Certaines personnes me font grief d'avoir, de leur point de vue, plagié Aristote sans le citer nommément. Mais, selon moi, ces gens ne comprennent pas que l'approche de la philosophie que j'ai privilégiée dans l'enseignement aux enfants exclut des références explicites. Par ailleurs, si je devais écrire une dizaine de nouvelles supplémentaires, nul doute que je m'inspirerais d'autres philosophies et que je tenterais de leur conférer une égale importance. Il reste qu'on conviendra que les idées de Platon et d'Aristote ne sont pas parmi les moindres.

En ce qui concerne les personnes qui sont chargées de la formation des enseignants pour le programme, nous sommes en train de rédiger une bibliographie exhaustive. Autrement dit, pratiquement ligne par ligne, nous indiquerons les sources et les références philosophiques ou parfois psychologiques et pédagogiques. Mais ce sera à l'usage exclusif de ces personnes et non pour les enseignants qui pourraient alors être tentés de faire des cours traidtionnels, ce qui est contraire à notre méthode, du moins pour l'enseignement de la philosophie aux enfants.

CEPH. La découverte par Harry de lois logiques comme résultat d'une recherche et non d'un apprentissage scolaire relève-t-elle d'une méthode socratique ? Par méthode socratique, j'entends que philosopher pourrait être : réfléchir sur ce qu'on ne sait pas.

M.L. En fait, je suis intéressé par la caractérisation que vous faites du socratisme et $j$ 'aurais tendance à y réfléchir moi-même de telle sorte que je risquerais de ne pas être en mesure de répondre maintenant à votre question. Peut-être devrais-je tout simplement mettre cela de côté et tenter de répondre directement à la question qui concerne la découverte par Harry de ce que vous appelez les lois de la logique. Je ne suis pas sûr qu'Harry découvre les lois de la logique. On peut se demander ce qu'Harry découvre par lui-même. Découvre-t-il le principe de conversion des propositions ou découvre-t-il le principe de validité ? Découvre-til toutes les lois de la logique? Par exemple, découvrir que certaines phrases restent vraies si elles proviennent d'autres phrases 
considérées comme prémisses, c'est beaucoup plus que le principe de conversion. De plus, Lisa (un autre personnage de la nouvelle) peut revendiquer une partie des découvertes.

Je dirais qu'à mon sens il y a beaucoup plus que le socratisme dans Harry. Il y a l'idée de Bruner que l'on peut enseigner n'importe quelle théorie à n'importe quel niveau ; cette notion brunerienne de la contextualisation selon laquelle les enfants apprennent mieux lorsque des principes sont intégrés à une histoire qu'ils peuvent comprendre. Mais Dewey l'avait déjà écrit dans son livre The child and the curriculum en 1903. Il y a aussi la théorie de Vico selon laquelle on n'apprend qu'à travers une mise en situation, une réactualisation.

Je considère ces précisions très importantes, car Dewey n'avait aucune prédilection pour Aristote et je pense qu'il s'en écartait beaucoup. Mais dans les leçons que Dewey a données en Chine et que l'on a redécouvertes récemment, Dewey dit que l'invention de la logique formelle par Aristote est peut-être le plus grand fait intellectuel dans l'histoire de l'humanité.

Quant à nous, c'est certainement un objectif que nous estimons valable que les enfants étudient, apprennent, tirent profit de ces grands faits intellectuels de l'humanité ; mais, lorsque nous essayons de réaliser cet objectif, nous nous apercevons que les enfants ne peuvent pas comprendre ces grandes œuvres. Une solution consisterait à en simplifier l'exposition, comme dans des manuels scolaires élémentaires. Mais la solution que nous estimons préférable serait de se demander : qu'a pu être un philosophe dans son enfance ? Et qu'a-t-il pu penser à cet âge, lui qui, vingt ou trente ans plus tard, inventera le système de la logique ? Ou, qu'était Galilée enfant? Qu'était Shakespeare enfant ? Donc, si l'on enseigne une théorie dans l'esprit dans lequel elle a été découverte, si l'on peut enseigner dans l'esprit de la découverte, on se trouvera à animer l'enfant dans la classe de la même excitation qu'a dû éprouver l'auteur de la découverte. Ce serait merveilleux, ce serait ce que Vico appelait "reenactment". Je pense que c'est possible.

Il y a bien des questions que l'on peut se poser à propos de cette sorte de reconstruction. Une critique consisterait à dire que nous n'avons pas le droit d'attribuer à des enfants des idées 
qu'ils n'avaient peut-être pas à leur âge. En fait, comment saurions-nous qu'Aristote, à l'âge de 12 ans, avait la moindre idée de ce que nous appelons aujourd'hui la logique aristotélicienne ? Évidemment, nous ne le savons pas, mais, par ailleurs, nous parlons bien de Socrate qui n'a jamais écrit et dont nous ne connaissons pas vraiment la vie en détail. Cependant nous savons que beaucoup de savants et de personnes qui ont contribué de façon éclatante à l'histoire de la pensée ont développé leurs idées à partir d'intuitions qu'ils ont eues plus tôt dans leur vie et souvent dans l'enfance. Ceux, parmi les savants, qui sont honnêtes, le reconnaissent volontiers ; combien d'autres se seraient sentis dévalorisés en se livrant à de pareilles confidences? Je pense que, lorsque, adultes, nous nous refusons à reconnaître ce que nous avons fait nous-mêmes étant enfants, nous enlevons quelque chose à l'enfance. On pourrait dire qu'il s'agit d'une exploitation intellectuelle de l'enfant. C'est du même ordre que de refuser de reconnaître ce qu'une femme peut réaliser sous prétexte que c'est une femme. Notre programme cherche effectivement à libérer l'enfant de cette prison, du secret où est tenue l'enfance.

CEPH. L'investigation de groupe qui est la méthode privilégiée par les enfants de votre nouvelle, correspond-elle à un souci de fonctionnement démocratique conformément aux idées de Dewey?

M.L. Je considère le programme d'enseignement de la philosophie aux enfants dans la mouvance de la pensée de Dewey. Certainement, la notion de démocratie chez Dewey, et la continuation de la démocratie dans l'éducation, est une préoccupation majeure de notre programme de philosophie pour enfants. Même s'il est vrai que Dewey n'a jamais explicitement parlé de philosophie pour enfants.

CEPH. Nous pensons que c'est une très bonne idée de mettre les enfants en situation par l'intermédiaire d'une nouvelle qui fait référence à leur situation dans la classe, à la maison, dans les relations avec d'autres enfants et avec des adultes, de telle sorte qu'ils apprennent à réfléchir à partir de la vie quotidienne. Également, nous apprécions le fait que, dans la nouvelle, on ne parvienne pas toujours à une conclusion et que les questions restent en général ouvertes. 
S'agit-il là de moyens pour atteindre un but que l'on pourrait définir comme celui de donner aux enfants le goût de la pensée, le goût de la réflexion?

M.L. Je pense que nous ne donnons pas plus aux enfants le désir de penser que nous pouvons leur donner le goût ou le désir d'être actifs dans un terrain de jeux. Je veux dire qu'ils ont déjà un tel désir ; penser est une activité qu'ils pratiquent déjà, que nous le voulions ou pas. Un philosophe, auquel on demandait comment obtenir qu'un enfant arrête de penser, répondit : tuezle ! (rires . . .) Notre but pourrait être de stimuler leur pensée, de les entraîner à penser mieux.

$\mathrm{CEPH}$. Ce qui m'amène à vous demander : le souci d'application de la pensée à la vie quotidienne, par exemple qu'Harry applique ses découvertes à tenter de résoudre les problèmes que lui pose l'épisode du caillou qui lui est lancé, ou à résoudre le problème de la mésentente entre Tony et son père, le souci que la pensée soit utile dans la vie quotidienne, pourrait-on dire qu'il relève d'une tendance au pragmatisme?

Si c'est le cas, que devient la stimulation d'une pensée qui serait plus théorique, plus gratuite? D'une pensée qui pourrait être de l'ordre de « penser pour penser »?

M.L. Le genre de paradigmes de pensée que Dewey développe : instigation à la recherche, vérification d'hypothèses, résolution de problèmes, destruction des obstacles etc., nous en avons certainement une reproduction dans la nouvelle. Le premier chapitre est une sorte de paradigme de recherche, il y a une initiation à l'hypothèse et à la vérification, et puisque cette première hypothèse est infirmée il y a élargissement de l'hypothèse, l'inclusion des propositions universelles négatives, la découverte d'applications etc . . Ceci, évidemment, correspond aux notions développées par Dewey et d'autres. Mais c'est également l'idée d'encourager la pensée en montrant que penser est une activité qui, parfois, permet de réussir ; que la pensée fonctionne effectivement dans le monde. Je veux dire que les enfants sont cloués à leur banc d'école jour après jour, mois après mois, année après année, et qu'on ne leur montre jamais d'application de ce qu'on Jeur enseigne qui soit d'un usage pratique dans leur vie quotidienne. Je ne dirais pas que ce soit une règle à observer dans 
tous les cas, mais que l'on devrait parfois leur montrer l'utilité de ce qu'ils apprennent.

Par ailleurs, il y a bien des cas où la pensée ne réussit pas, où il y a des impasses, des échecs et où Harry ressent de la frustration et blâme Lisa etc . . . La pensée ne réussit pas toujours, mais parfois.

CEPH. Estimez-vous qu'il 'est important de maintenir le mot " philosophie " dans le programme de philosophie pour enfants ou serait-il indifférent d'appeler cela, par exemple dans le cas de Harry, "logique pour enfants " ou "analytic teaching" ?

M.L. Je ne pense pas du tout qu'il soit correct de nommer ce programme autrement que par le mot " philosophie ". À moins qu'il y ait des raisons majeures et extérieures qui exercent une contrainte en ce sens. C'est le cas au Texas. Par ailleurs, nommer le tout du nom de la partie, comme dans le cas de "logique pour enfants " serait une aberration. Notre intérêt est dans la philosophie qui, quoi qu'en pensent certaines personnes, est une discipline et l'un des plus importants domaines d'étude.

Il y a bien, je pense, dans la tradition britannique positiviste et empiriste une tendance à dénier à la philosophie une existence séparée et à prétendre qu'elle peut tout au plus être la critique d'autres disciplines particulières qui, elles, existent par elles-mêmes. Il y aurait ainsi une philosophie des sciences qui ne traiterait que de certains concepts relatifs aux sciences, mais il n'y aurait pas telle chose qu'une philosophie en soi. II y aurait bien des "philosophies de . . ." mais pas de philosophie tout court ; c'est-à-dire des philosophies qui auraient pour objet d'autres disciplines mais pas de philosophie qui aurait pour objet le monde lui-même et serait une discipline autonome. Or, je pense que la philosophie comprend ces deux aspects et que les positivistes se trompent.

CEPH. Est-ce que chacune des nouvelles du programme (Harry, Pixie, etc,) est un tout en soi, une entité à part ? Ou bien estce que vous voyez une progression dans la formation philosophique à travers la succession des nouvelles?

M.L. Je dirais que les enfants qui suivent l'ensemble du programme deviennent de plus en plus compétents en philosophie 
au fur et à mesure qu'ils étudient les nouvelles et peuvent mettre en application ce qu'ils ont appris précédemment dans l'étude d'une nouvelle subséquente. C'est-à-dire que les trois derniers livres représentent des applications des capacités, de la compréhension, des approches qui sont développées dans les livres précédents. Je pense donc que, oui, les enfants font une progression, comme, en passant, les adultes en font une dans la nouvelle. Par exemple le père de Harry change d'idée et fait des progrès ... .

CEPH. J'aurais maintenant quelques questions plus précises à vous poser sur la nouvelle Harry, si vous le voulez bien. L'épisode du salut du drapeau (le cas d'un enfant qui refuse de se conformer à cette pratique, les discussions passionnées sur le droit à la dissidence, l'appartenance à une communauté etc . . .) tient une place importante dans Harry. Or, dans de nombreux pays, on ne retrouve pas une telle pratique qui n'a donc pas de signification concrète pour les enfants. Avez-vous pensé à une solution de remplacement, éventuellement, pour la traduction et l'adaptation?

M.L. Dans certains pays, cet épisode ne pose pas de problème, même si la pratique de saluer le drapeau n'y existe pas. Les enfants comprennent que c'est la pratique aux États-Unis et peuvent réfléchir sur le problème posé dans la nouvelle.

"Quant à moi, j'aurais préféré pouvoir écrire la nouvelle de telle sorte que l'on ne sache pas que cela se passe aux États-Unis et que chaque nouvelle puisse être traduite de façon à émaner de la culture du pays. À terme, c'est un objectif souhaitable, d'éliminer cet aspect "américain » de la nouvelle. Je crois que les exemples peuvent effectivement être changés pour s'adapter à la culture locale. Il y a ainsi de multiples façons d'illustrer le principe de la conversion des propositions ; dans les versions du programme en langue arabe, les illustrations se réferent aux insectes qui piquent, ailés ou non ; c'est le cas en Égypte, et je n'ai aucune objection, au contraire.

Quant à cet épisode du drapeau, je crois qu'il faut reconnaître le principe qui est en jeu. Ce principe est de confronter deux autorités aussi respectables que contradictoires quant au comportement de l'enfant qui les reconnaît toutes deux (dans 
ce cas, il s'agit de la famille et de l'école, de l'état et de l'église). Il s'agit de montrer que cette confrontation tragique n'a pas de solution rationnelle qui, du moins s'imposerait à tout coup. En ce sens, le chapitre dix est l'histoire d'un échec, les enfants le ressentent ainsi, ils ne peuvent parvenir à une solution. Pour adapter, on pourrait imaginer quelque chose comme la situation d'un enfant dont le père dit : " peu importe ce que l'école t'enseigne comme comportement social, légal, moral ; si quelqu'un te menace, tu dois te battre, tu dois te défendre toi-même ». Et le principal de l'école dirait à l'enfant : « peu importe la menace, tu ne dois pas te battre ; dans une société qui est régie par la loi, on ne doit pas se faire justice soi-même mais soumettre le problème aux autorités compétentes ». Cependant, en l'absence de ces autorités, quelqu'un pourrait s'estimer en droit de prendre les mesures nécessaires à sa propre protection. Cela, on le sait, arrive dans le métro, par les temps qui courent. Mais c'est un réel problème pour des enfants qui se demandent s'ils peuvent se défendre eux-mêmes, alors que leur père répondrait oui et le principal d'école non. Voici donc une autre façon d'illustrer une telle confrontation ; cependant je pense que l'on pourrait en trouver bien d'autres.

$\mathrm{CEPH}$. Cette question sera plus générale : votre programme évitet-il le plus possible une prise de parti politique ou idéologique ?

M.L. Mon programme d'enseignement de la philosophie aux enfants ne prend pas parti, mais je pense qu'il y a une prise de position qui se dégage de l'ensemble. Je veux dire qu'il me paraît improbable de lire les livres qui le constituent sans remarquer une prédilection pour les valeurs démocratiques, une défense de l'individu, une attaque contre le conformisme et l'autoritarisme, une défense du droit à questionner, du droit à penser, du droit à l'éducation, du droit de participer. Je pense qu'il y a certains types de valeurs auxquelles aucun personnage ne s'oppose dans la nouvelle, et qui, s'il y a opposition, sont défendues par les enfants mis en scène. C'est donc la manifestation de ce que l'on pourrait appeler une idéologie sous-jacente, implicite.

CEPH. La remise en question de préjugés, comme le racisme, l'ethnocentrisme, peut-elle aller jusqu'à laisser se développer ce que l'on pourrait appeler un préjugé inverse ? Ainsi, dans Harry, 
une enfant noire dit qu'en Afrique on partage tout, au contraire de l'Occident ; "lorsque la famine s'installe, tout le monde a faim ...."

$\mathrm{Ne}$ pensez-vous pas qu'il faudrait relativiser une telle conception, non pas dans la nouvelle car cette enfant exprime son opinion, mais dans le livre du maître, par le moyen d'exercices ou d'explications ? Par exemple, on pourrait parler de la structure sociale de certains pays du Tiers-Monde, et l'Afrique n'y fait pas exception; des inégalités économiques . . .

M.L. Ce serait une bonne chose de le faire. Il y a eu jusqu'ici quatre différentes versions du manuel de Harry, j'aimerais que l'actuelle soit la dernière, mais, vous le voyez, nous découvrons de nouveaux problèmes.

Cependant, dans la première version, nous avions l'ambition d'établir des ponts avec d'autres disciplines; le manuel devenait de plus en plus chargé et il ne restait plus de place pour la philosophie elle-même. Aussi bien, nous avons abandonné cette façon de faire. L'exemple que vous citez dans votre question est-il un préjugé inverse ? Cela me fait penser à l'action positive, s'agit-il d'une discrimination? Remarquons que la nouvelle met en scène deux enfants noirs qui ont des comportements, des attitudes extrêmement différents : l'une prend fait et cause pour ses " racines ", l'autre est plutôt occidental. On peut donc discuter la question de savoir si c'est en tant que noire que l'enfant exprime cette opinion sur la Tanzanie. Et, quand bien même ce serait le cas ? Des images différentes des noirs sont tellement prévalentes que celle de l'enfant dont vous parlez vaut la peine d'être exposée. Bien sûr, cela peut être discuté dans la classe. Vous savez que les enfants émettent toutes sortes de théories sur le monde, sur leurs ancêtres, sur l'histoire. C'est le cas d'enfants noirs américains, comme d'enfants d'origine italienne etc . . . Quant à moi, je pense qu'il faut combattre tous les préjugés et que le meilleur moyen de le faire est de développer des capacités d'analyse rationnelle; il y a plein d'exercices de ce genre dans Harry lorsque nous montrons que certains syllogismes portent préjudice à un groupe et que c'est incorrect.

CEPH. Dans Harry apparaît la définition de l'être humain comme un être de culture. Dans quel sens, si on prenait au sérieux cette 
définition qui apparaît au cours d'une discussion, devrait-on entendre le mot « culture »?

M.L. Il s'agit de la notion anthropologique de la totalité des œuvres et non seulement des grandes œuvres. C'est plutôt une notion hégélienne, la culture comme esprit objectif, et non pas la culture comme haute culture, grandes œuvres d'art, etc.

CEPH. Nous abordons maintenant la dernière partie de cette entrevue. Pourrions-nous parler, plus spécifiquement, de la formation des enfants ? Ainsi, nous avons tous été intéressés par la remarque que les capacités en lecture et en mathématiques des enfants qui suivent le programme de philosophie sont nettement améliorées. Votre équipe a pratiqué des expérimentations, des tests, qui le démontrent, et c'est certainement un résultat qui est remarquable.

L'amélioration des capacités des enfants en lecture et en mathématiques constitue-t-elle un objectif majeur de votre programme?

M.L. Non, mais c'est une bonne chose. Certaines institutions scolaires y voient la raison majeure pour laquelle elles implantent le programme. Dans ce cas, peut-être pourrait-on dire qu'elles agissent dans le bon sens, mais pas pour la bonne raison. On peut espérer qu'après l'implantation du programme, il en soit autrement. Par ailleurs, il est vrai que, dans les institutions scolaires, on parle de plus en plus de développer la pensée et la pensée critique. Je crois qu'en effet le répertoire des méthodes d'éducation traditionnelles était à bout de souffle dans les années cinquante ; on s'est aperçu que la formation de l'enfant ne serait pas améliorée en ajoutant des heures de classe ou en payant plus cher les enseignants.

Dans les années soixante, on a eu recours à une éducation de type affectif, c'était la mode : créativité, thérapie, dynamique de groupe ; le tout dans le but de venir en aide aux enfants des classes moyennes, de leur permettre de surmonter leurs inhibitions. Cette mode eut un effet destructeur, ou autodestructeur ; ce type d'éducation ne menait nulle part. Par contre, dans les années soixante-dix, il y eut un mouvement de retour vers l'éducation traditionnelle; ce fut, à mon avis un 
mouvement réactionnaire, qui n'avait rien d'innovateur, et qui reproduisait les mêmes approches et les mêmes erreurs que dans le passé. Depuis, donc, une trentaine d'années le système d'éducation cherche désespérément des solutions, une innovation utile. C'est dans cette perspective qu'il faut considérer l'engouement actuel pour la pensée, pour un apprentissage qui ne se réduise pas à la transmission de connaissances. Il s'agit d'une immense opportunité pour la philosophie : la philosophie est certainement la discipline qui serait le mieux à même de répondre intelligemment à ce besoin.

Malheureusement, la plupart des philosophes n'ont pas pris garde à ces mouvements dans l'éducation, ils n'ont pas tendance à intervenir, ils ont pris la fâcheuse habitude d'avoir les mains propres et de ne pas se risquer dans le domaine de l'éducation des enfants. Je pense, j'espère qu'ils s'impliqueront davantage. Un groupe comme le vôtre, à la Société de Philosophie du Québec, représente une lueur d'espoir, un signe de changement d'attitude. Je crois profondément que l'implication des philosophes, de la philosophie, dans l'éducation des enfants est une voie d'avenir, dans le monde entier ; à l'exception peut-être de certains pays totalitaires.

CEPH. On sait que Descartes soulignait que le syllogisme lui paraissait stérile et servir plutôt à exposer ce qu'on sait déjà qu'à découvrir du nouveau. Dans la mesure où la logique joue un rôle d'importance dans Harry, comment voyez-vous, vous-même, le rôle formateur de la logique?

M.L. Je pense qu'effectivement, dans le langage par exemple, on ne bâtit pas une phrase en se référant consciemment et explicitement aux règles de la grammaire ; la syntaxe est ce qui nous permet, naturellement, de construire de longues phrases presque instinctivement. C'est la même problématique pour la logique ; nous n'en avons pas une claire conscience alors même que nous l'utilisons. Il est vrai que l'on rencontre très rarement un syllogisme formel dans la conversation. De sorte qu'il pourrait bien ne pas y avoir d'intérêt pratique à l'usage conscient de la logique.

Mais, d'une part, nous ressentons un besoin de rechercher la cohérence lorsque nous parlons, de nous assurer que nous ne nous contredisons pas dans la même phrase ; ce n'est pas que 
nous ayons clairement en tête les lois de la logique, mais nous sentons que ce que nous disons n'aurait pas de sens autrement. D'autre part, il y a un rôle symbolique de la logique dans l'éducation : l'enfant, en troisième ou quatrième année, est probablement sceptique quant à la valeur des arguments de différentes personnes ; il est en danger de devenir subjectiviste, nihiliste; relativiste ; il aura tendance à croire que toutes les opinions se valent et que l'on peut soutenir n'importe quoi. La découverte qu'il n'est pas vrai que l'on puisse dire n'importe quoi, que l'on puisse conclure de n'importe quelle façon, cette découverte est la révélation d'une certaine objectivité, aussi impressionnante, en un sens, que celle qui est en jeu dans la physique à propos du monde extérieur. Découvrir qu'il y a de petits paradigmes, de petites règles qui permettent de préserver la vérité d'une proposition par rapport à l'autre, est une sorte d'assurance de stabilité dans un monde intellectuel qui serait autrement dans les limbes. C'est une protection contre le genre de cynisme qu'ils manifestent parfois, non parce qu'ils sont cyniques, mais parce qu'ils nous lancent le défi de leur prouver qu'ils n'ont pas raison de le devenir. La logique semble être la meilleure arme que nous ayons contre ce genre de cynisme ou de relativisme ; procuronsla aux enfants. Ce n'est pas la seule arme, je pense que la science en est une autre. Tel est, selon moi, l'enjeu de la formation logique.

CEPH. Une dernière question : comment voyez-vous, idéalement, la formation philosophique des enseignants qui auraient à utiliser votre programme de philosophie pour enfants ?

M.L. Dès la conception, dès la naissance (rires).

C'est évidemment un problème très difficile. L'on pourrait se décourager et perdre tout espoir si l'on devait arriver à la conclusion que les seules personnes compétentes pour enseigner ce programme devraient avoir un doctorat de philosophie. Ce serait peut-être là l'idéal, mais il est évident que ce programme n'aurait pas d'avenir, ce serait utopique.

Puisque l'engagement de nouveaux enseignants est pratiquement nul, nous avons choisi la voie de proposer un perfectionnement, une formation supplémentaire à l'intention des enseignants en fonction (qui, dans leur immense majorité, n'ont 
pas étudié la philosophie). Pour implanter le programme, la seule solution praticable est de le faire avec les enseignants qui sont en place à l'élémentaire et au secondaire. Les grands changements pourraient être dans les programmes de formation des enseignants. Traditionnellement, à un extrême, un bon professeur serait celui qui connaît sa matière ; à l'autre extrême, vers lequel $j$ 'inclinerais personnellement, on pourrait argumenter qu'il y a un grand nombre de professeurs qui connaissent parfaitement leur matière et qui n'obtiennent pas davantage de résultats dans l'éducation que ceux qui seraient plus ignorants. Enseigner, c'est, pour une grande part, communiquer et non murmurer en écrivant au tableau devant un public qui s'ennuie ou qui dort.

Bien sûr, ne pas connaître sa matière n'est pas, non plus, une garantie de succès. Mais le cas de la philosophie me semble différent : si l'enseignant peut devenir un membre d'une communauté de recherche, s'il peut découvrir la philosophie en même temps que les étudiants, le besoin d'équiper cet enseignant d'une grande culture philosophique serait beaucoup moindre.

Notre façon de faire est d'insister d'abord sur la méthode d'enseignement du programme et ce n'est que lorsque l'enseignant en formation ou en perfectionnement développe une curiosité concernant les sources, les références philosophiques, que l'on peut les lui fournir à ce moment-là dans la mesure où il adopte l'attitude professionnelle de garder ces théories (celle de Platon, de Spinoza etc.) pour lui-même sans les imposer aux enfants. Je ne dis pas qu'il ne pourrait y avoir certains enfants qui tireraient profit d'un enseignement traditionnel de la philosophie ; il ne s'agit pas d'enfants surdoués mais il arrive que des enfants mûrissent très jeunes ; c'est cependant rare, et l'on ne peut se baser sur eux pour bâtir un programme. D'ailleurs ils en tireraient profit plus vite, pas mieux. 


\section{BIBLIOGRAPHIE}

LIPMAN, Matthew et al., Philosophy in the Classroom, Philadelphia, Temple University Press, 1980.

LIPMAN, Matthew et SHARP, Ann Margaret, Growing up with Philosophy, Philadelphia, Temple University Press, 1978.

LIPMAN, Matthew, La Découverte d'Harry (Harry Stottlemeier's Discovery), traduction et notes par Pierre Belaval, Paris, Vrin.

LIPMAN, Matthew, Pixie, traduction française Arsène Richard, Les Éditions d'Acadie ltée, 1984.

REED, Ronald F., Talking with Children, Denver (Colorado), Arden Press Inc., 1983.

En plus de ces ouvrages, on peut également commander les cinq nouvelles suivantes ainsi que les livres d'instruction d'accompagnement à :

The First Montain Foundation,

P.O. Box 196,

Montclair, New Jersey. 07042.

Harry Stottlemeier's Discovery. Philosophical Inquiry : Instructional Manual to accompany Harry Stottlemeier's Discovery.

Lisa. Ethical Inquiry : Instructional Manual to accompany Lisa.

Suki. Writing - How and Why: Instructional Manual to accompany Suki.

Mark. Social Inquiry : Instructional Manual to accompany Mark.

Pixie. Looking for Meaning : Instructional Manual to accompany Pixie.

Mentionnons enfin pour terminer :

The Philosophy for Children Newsletter, publié 6 fois par année par The First Moutain Foundation. 